\title{
An example of phase holonomy in wKB theory
}

\author{
Michael Wilkinson \\ H H Wills Physics Laboratory, Royal Fort, Tyndall Avenue, Bristol, BS8 1TL, UK $\dagger$
}

Received 4 June 1984

Abstract. This paper discusses the application of WKB theory to Harper's equation

$$
\psi_{n+1}+\psi_{n-1}+2 \alpha \cos (2 \pi \beta n+\delta) \psi_{n}=E \psi_{n} \text {, }
$$

in the case in which $\beta$ is very close to a rational number, $p / q$.

The WKB wavefunction for this system is a vector valued quantity, proportional to an eigenvector $\boldsymbol{u}$ of a matrix $\hat{H}(x, p)$, which is parametrised by the phase space coordinates $x$ and $p$. The complex phase of $\boldsymbol{u}$ is determined by a non-holonomic connection rule; when transported around a cycle and in phase space, $\boldsymbol{u}$ is multiplied by a phase factor $\mathrm{e}^{1 \%}$. This phase change manifests itself as a modification of the Bohr-Sommerfeld quantisation condition.

\section{Introduction}

This paper describes an unusual form of Bohr-Sommerfeld quantisation, involving a holonomy argument. As well as being interesting in its own right, the method discussed here can be applied to the difficult problem of finding the Bohr-Sommerfeld quantisation condition for Bloch electrons in a magnetic field. The system treated in this paper is a simplified model for this problem, which is often called Harper's equation. This model will be introduced in $\$ 2$; the remainder of this introduction will describe the principle of the method.

For the system considered, the wKB wavefunction can be thought of as a vectorvalued quantity, given by

$$
\boldsymbol{\psi}(x)=A(x) \boldsymbol{u}(x) \exp \left(\frac{\mathrm{i}}{\hbar} \int^{x} p\left(x^{\prime}\right) \mathrm{d} x^{\prime}\right)
$$

where $p(x)$ and $\boldsymbol{A}(x)$ are slowly varying functions, and the vector $\boldsymbol{u}$ is a solution of the eigenvalue equation

$$
\hat{H}(x, p) \boldsymbol{u}=\varepsilon \boldsymbol{\varepsilon} .
$$

In equation (1.2), $\varepsilon$ is the energy of the solution $\psi(x)$, and $\hat{H}$ a complex Hermitian matrix which is a function of two parameters $x$ and $p$. Since the energy $E=\varepsilon(x, p)$ is a constant for a given solution, equation (1.2) gives both $\boldsymbol{u}$ and $p$ as functions of $x$, as in (1.1). The curves in the $x-p$ plane defined by $E=\varepsilon(x, p)=$ constant are called phase trajectories. When the phase trajectories given by (1.2) are closed orbits, then a solution $\psi(x)$ must remain single-valued when it is traced around the phase trajectory.

† Address after September 1st 1984: Department of Physics, California Institute of Technology, Pasadena, California 91125 , USA 
This condition is only satisfied for certain values of $E$, which are determined by a Bohr-Sommerfeld quantisation condition.

In equation (1.2), the eigenvector $\boldsymbol{u}(x, p)$ is determined only up to a complex-valued multiplying constant, or, if $\boldsymbol{u}$ is assumed to be normalised, up to a complex phase factor $\mathrm{e}^{\mathrm{i} \theta}$. This phase factor can be determined by requiring that the amplitude $A(x)$ in (1.1) be real. Given this condition on $A(x)$, it will be shown how the wKB theory for the system leads to a connection formula, by means of which the vector $\boldsymbol{u}$ can be transported through the phase space with its phase fully determined. It turns out that this phase connection is non-holonomic, so that when $\boldsymbol{u}$ is transported clockwise around a closed circuit in phase space, it is multiplied by a phase factor $e^{i \gamma}$.

This phase factor affects the Bohr-Sommerfeld quantisation condition. Consider the phase change of the solution (1.1) after making one circuit of a closed phase trajectory. This has contributions from the oscillatory term, from a pair of turning points where $p(x)=0$ and $A(x)$ diverges, plus a contribution $\gamma(E)$ from the phase factor evaluated for a phase trajectory of energy $E$. The condition for the wavefunction $\psi$ to be single valued is therefore

$$
2 \pi n=\frac{1}{\hbar} \oint_{\varepsilon=E_{n}} p(x) \mathrm{d} x+\frac{\pi}{2}+\frac{\pi}{2}+\gamma\left(E_{n}\right),
$$

or

$$
\oint_{\varepsilon=E_{n}} p(x) \mathrm{d} x=\left[2 \pi\left(n+\frac{1}{2}\right)-\gamma\left(E_{n}\right)\right] \hbar .
$$

This equation (1.4) is the Bohr-Sommerfeld quantisation condition determining the eigenvalues $E_{n}$ of the system.

The plan of this paper is as follows. Section 2 introduces the system under consideration and discusses how wKB theory can be applied to this system. Section 3 derives an asymptotic formula for the product of a string of slowly varying transfer matrices. Section 4 applies this formula to the wKB problem for Harper's equation, and $\S 5$ obtains the Bohr-Sommerfeld quantisation condition. Section 6 summarises the theoretical results and compares them with numerical values, and $\S 7$ discusses the connections between this work and recent work on adiabatic theory and the quantised Hall effect.

\section{WKB analysis of Harper's equation}

The system analysed in this paper is Harper's equation

$$
\psi_{n+1}+\psi_{n-1}+2 \alpha \cos (2 \pi \beta n+\delta) \psi_{n}=E \psi_{n},
$$

which is frequently used in models for Bloch electrons in a magnetic field, and as a model for electrons in an incommensurate potential (Harper 1955, Simon 1982). As pointed out by Sokoloff (1981), solutions of (2.1) can be obtained by a wKB method whenever $\beta$ is sufficiently close to a rational number, $p / q$ (where $p$ and $q$ are coprime integers). The condition for WKB theory to be applicable is

$$
\left|q^{2} \Delta \beta\right| \ll 1, \quad \Delta \beta=\beta-p / q,
$$

and for almost all $\beta$, there exist values of $p / q$ for which $\left|q^{2} \Delta \beta\right|$ is arbitrarily small. This follows from a property of continued fractions (Khinchin 1964). 
Before describing how wкв theory can be applied to (2.2), it will be useful to consider the case $\beta=p / q$, so that the coefficients of the difference equation (2.1) are periodic with period $q$. In this case, therefore, Bloch's theorem applies and exact solutions can be obtained; the Bloch solution has the form

$$
\psi_{n}=\mathrm{e}^{i k n} u_{n}(\delta, k)
$$

where $u_{n}$ is periodic with period $q$

$$
u_{n+q}=u_{n} \text {. }
$$

This result can also be written in terms of a set of Fourier amplitudes for $u_{n}$

$$
\psi_{n}=\mathrm{e}^{\mathrm{i} k n} \sum_{m=0}^{q-1} a_{m} \exp (2 \pi \mathrm{i} p m n / q),
$$

which will prove more useful for some purposes.

From equation (2.1), it can be seen that the $q$-component vectors $u_{n}$ or $a_{m}$ can be determined as eigenvectors of a $q \times q$ complex Hermitian matrix. To distinguish these matrices and vectors from some two-component vectors and $2 \times 2$ matrices which will be introduced later, a quantum mechanical notation will be used;

$$
\hat{H}(\delta, k)|u(\delta, k)\rangle=\varepsilon|u(\delta, k)\rangle,
$$

where the matrix elements of $\hat{H}$ are given by

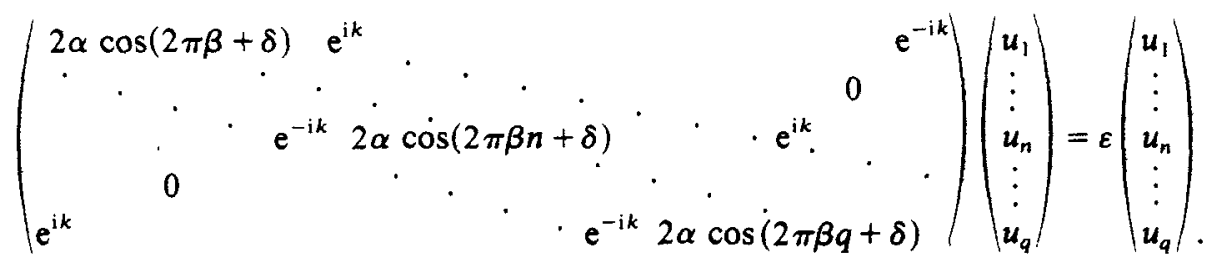

It is fairly easy to show that the eigenvalues $E$ are periodic in both $\delta$ and $k$ with period $2 \pi / q$; in fact the $q$ eigenvalues are given by the equation

$$
f(E)=\cos q k+\alpha^{q} \cos q \delta,
$$

where $f$ is a $q$ th degree polynomial (Wilkinson 1984). The $q$ different sheets of $\varepsilon(\delta, k)$ normally do not touch each other. When $q$ is even, however, one pair of sheets of $\varepsilon(\delta, k)$ does touch at isolated points in the $\delta-k$ plane (Bellissard and Simon 1982).

There is another, complementary, method for analysing equation (2.1) when $\beta$ is rational (i.e. $\Delta \beta=0$ ); this is the transfer matrix method. It is easy to see that equation (2.1) can be written in the form

$$
\left(\begin{array}{c}
\psi_{n+1} \\
\psi_{n}
\end{array}\right)=\tilde{T}\left(x_{n}, E\right)\left(\begin{array}{c}
\psi_{n} \\
\psi_{n-1}
\end{array}\right),
$$

where

$$
\begin{aligned}
& \tilde{T}(x, E)=\left(\begin{array}{cc}
E-2 \alpha \cos x & -1 \\
1 & 0
\end{array}\right), \\
& x_{n}=2 \pi \beta n+\delta .
\end{aligned}
$$


Consider a transfer matrix $\tilde{M}(x, E)$ describing a 'jump' of $q$ steps

$$
\left(\begin{array}{c}
\psi_{(n+1) q+1} \\
\psi_{(n+1) q}
\end{array}\right)=\tilde{M}\left(x_{n}, E\right)\left(\begin{array}{c}
\psi_{n q+1} \\
\psi_{n q}
\end{array}\right)
$$

where now

$$
\begin{aligned}
& \tilde{M}(x, E)=\tilde{T}[x+(q-1) \beta, E] \ldots \tilde{T}(x+\beta, E) \tilde{T}(x, E), \\
& x_{n}=2 \pi \beta q n+\delta=2 \pi p n+\delta .
\end{aligned}
$$

Now the transfer matrix $\tilde{M}$ is independent of $n$. The eigenvalue condition on $E$ is then just that the eigenvalues of the transfer matrix $\tilde{M}$ lie on the unit circle. Since $\tilde{T}$, and therefore $\tilde{M}$, both satisfy

$$
\operatorname{det} \tilde{T}=\operatorname{det} \tilde{M}=1 \text {, }
$$

this condition becomes

$$
2 \cos k=\operatorname{Tr} \tilde{M}(\delta, E) .
$$

Having found the eigenvalues $E(\delta, k)$ using (2.14), the wavefunctions can be generated by means of the formula (2.9).

To summarise: there are two approaches to solving (2.1) when $\beta$ is rational; one, which will be termed the Bloch picture, involves solving a $q \times q$ Hermitean eigenvalue equation, the other, which will be termed the Floquet picture, involves considering products of $q 2 \times 2$ transfer matrices. The rest of this section will show how WKB methods can be applied when $\beta$ is close to a rational number. First the application of the WKB method within the Bloch picture will be described. This has previously been attempted by Sokoloff (1981); it cannot be carried through to yield a full solution, but is worth describing since it is easier to understand because it is closer to ordinary WKB methods. Finally, the application of the WKB method in the Floquet picture will be described. This is harder to visualise, but does lead to a full solution of the problem.

When $\Delta \beta$ is small, the solution must 'locally' look like a solution of the form (2.3). On a 'global' scale, however, there is a slow change in the phase parameter $\delta$; in the region of the amplitude $\psi_{n}$ the effective phase $\delta^{\prime}$ is

$$
\delta^{\prime}=\delta+n \hbar / q
$$

where

$$
\hbar=2 \pi \Delta \beta q \text {. }
$$

The symbol $\hbar$ is used in (2.15) because this quantity will be the small parameter of the WKB theory. In the neighbourhood of the amplitude $\psi_{n}$, the solution resembles a solution of the form (2.3) or (2.4) with $\delta$ replaced by $\delta^{\prime}$.

The Bloch wavevector, $k$, now varies slowly with $n$ : the energy $E$ is still given by equation (2.7), and is a constant for a given solution, so that (2.7) defines an implicit relationship between $k$ and $\delta$. The energy $E$ should now be considered to depend on $\hbar$ as well as $\delta$ and $k$,

$$
E=\varepsilon\left(\delta^{\prime}, k ; \hbar\right)=\varepsilon_{0}\left(\delta^{\prime}, k\right)+\hbar \varepsilon_{1}\left(\delta^{\prime}, k\right)
$$

since $\beta$ in (2.7) depends on $\hbar$. The term of order $\hbar$ in (2.17) will be important in what follows. 
Following Sokoloff (1981), equation (2.1) is written in the form

$\psi(x+\hbar / q)+\psi(x-\hbar / q)+2 \alpha \cos \left(2 \pi p x / \hbar+x-x_{0}\right) \psi(x)=E \psi(x)$,

where

$x_{0}=(2 \pi p \delta / q) \bmod 2 \pi, \quad \psi_{n}=\psi\left(x_{n}\right), \quad x_{n}=n \hbar / q+\delta$.

By comparison with equation (2.5), this suggests a trial solution of the form

$$
\psi(x)=A(x) \exp (\mathrm{i} S(x) / \hbar) \sum_{m=0}^{q-1} a_{m}(x) \exp (2 \pi \mathrm{i} p m x / \hbar)
$$

This trial solution corresponds to the abstract solution introduced in equation (1.1). The role of the vector $\boldsymbol{u}$ in $(1.1)$ is played by the set of Fourier coefficients $a_{m}$ in $(2.20)$. These coefficients are easily shown, by substituting $(2.20)$ into $(2.18)$, to satisfy the equation

$$
\alpha \mathrm{e}^{-\mathrm{i} x} a_{m+1}+\alpha \mathrm{e}^{\mathrm{i} x} a_{m-1}+2 \cos \left[\left(2 \pi p m+S^{\prime}\right) / q\right] a_{m}=E a_{m},
$$

which is an eigenvalue equation for $E$ corresponding to (1.2).

The next step in Sokoloff's approach to the wKB theory of Harper's equation is to expand $\psi(x \pm \hbar / q)$ in (2.20) in powers of $\hbar$, and insert the result into (2.18). Unfortunately, this does not lead to a consistent result; if the calculation is carried out correctly it is found that $q$ independent equations are obtained which $A(x)$ should satisfy. (The solution which Sokoloff obtains for $A(x)$ is easily found to be incorrect.)

It turns out that a full solution of the wKB problem can be obtained in the Floquet picture, however. The transfer matrices $\tilde{M}(x, E)$ introduced in $(2.11)$ are now no longer independent of $n$, but provided (2.2) is satisfied, these transfer matrices are at least slowly varying. It is possible to calculate the product of a string of slowly varying matrices;

$$
\begin{gathered}
\tilde{G}\left(x, x^{\prime} ; \hbar\right)=\tilde{M}_{E}(x, \hbar) \tilde{M}_{E}(x-\hbar, \hbar) \ldots \tilde{M}_{E}\left(x^{\prime}+\hbar, \hbar\right) \tilde{M}_{E}\left(x^{\prime}, \hbar\right), \\
\tilde{M}_{E}(x, \hbar)=\tilde{T}\{x+2 \pi p(q-1) / q+[(q-1) / q] \hbar, E\} \ldots \tilde{T}(x+2 \pi p / q+\hbar / q, E) \tilde{T}(x, E), \\
\tilde{T}(x, E)=\left(\begin{array}{cc}
E-2 \alpha \cos x & -1 \\
1 & 0
\end{array}\right) .
\end{gathered}
$$

A simple formula for the product $\tilde{G}\left(x, x^{\prime} ; \hbar\right)$ will be derived in $\S 3$.

Before going on to discuss the WKB theory in detail, it will be helpful to describe briefly the final results of the calculation.

Suppose that $\beta$ is a low denominator rational number, $\beta_{0}=p / q$. The spectrum then consists of $q$ bands (the centre two bands touch if $q$ is even), and $E$ is a periodic function of the Bloch wavevector $k$ and position parameter $\delta ; E=\varepsilon(\delta, k)$, with $q$ branches, one for each band.

When $\beta$ is close to $\beta_{0}, \beta=\beta_{0}+\hbar / 2 \pi q$, then wKB theory can be applied and $\delta$ and $k$ become the position and momentum coordinates of the phase-space $(\delta \rightarrow x, q k \rightarrow p=$ $\left.S^{\prime}\right)$. The dispersion relation $E=\varepsilon(\delta, k)$ for a given band becomes the classical Hamiltonian; $H(x, p)=\varepsilon(x, k q)$.

When the phase trajectories (contours of $E=H(x, p)$ ) are closed orbits, the energies of the eigenstates are restricted by a Bohr-Sommerfeld quantisation condition. Some contours of a typical $H(x, p)$ are shown in figure 1 for the case $\alpha=1$, when (by symmetry) all the phase trajectories are closed orbits. Each of the $q$ bands of the 


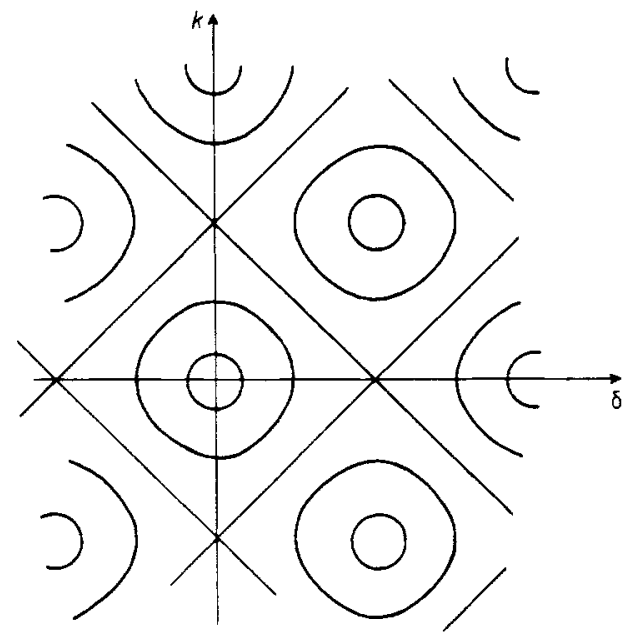

Figure 1. Phase trajectories of the classical Hamiltonian when $\alpha=1$. All the phase trajectories are closed orbits.

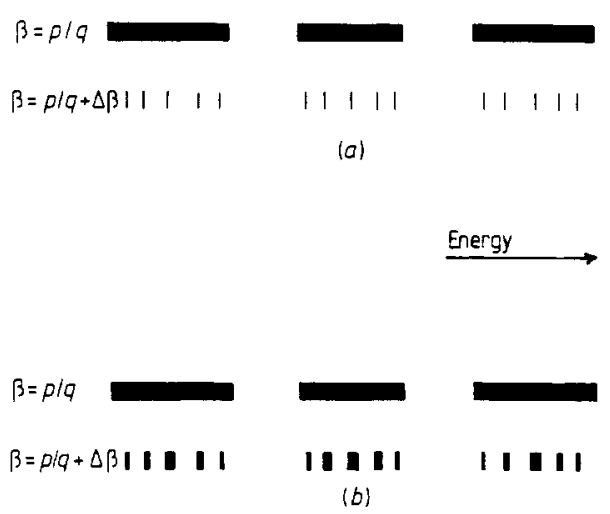

Figure 2. (a) Bloch bands of the commensurate system, $\beta=p / q$, become Bohr-Sommerfeld quantised levels when $\beta$ is changed by a small amount. (b) The Bohr-Sommerfeld quantised levels are in practice broadened slightly by tunnelling effects.

spectrum is then split into a number of Bohr-Sommerfeld quantised levels. This situation is shown schematically in figure $2(a)$.

These Bohr-Sommerfeld quantised levels are not truly discrete; since the classical Hamiltonian $H(x, p)$ is a periodic function of $x$ and $p$, the energy levels are broadened slightly by tunnelling between degenerate states, as illustrated schematically in figure $2(b)$. These tunnelling effects are discussed in detail in Wilkinson (1984) for the case $\beta_{0}=0$. Since the broadening of the levels due to tunnelling vanishes very rapidly as $\hbar \rightarrow 0$, as

$$
E_{\text {tunnelling }} \simeq \exp (- \text { constant } / \hbar),
$$

it does still make sense to obtain an asymptotic formula for the Bohr-Sommerfeld quantisation condition.

\section{Adiabatic matrix strings}

This section derives a formula for the product of a string of slowly varying matrices, $\tilde{M}$. An asymptotic formula is obtained for the product $\tilde{G}$, defined by

$$
\tilde{G}\left(x, x^{\prime} ; \hbar\right)=\prod_{\substack{n=0 \\ x^{\prime \prime}=x^{\prime}+n \hbar}}^{\mathcal{N}} \tilde{M}\left(x^{\prime \prime}, \hbar\right), \quad \mathcal{N}=\frac{\left|x-x^{\prime}\right|}{\hbar},
$$

i.e.

$$
\tilde{G}\left(x, x^{\prime} ; \hbar\right)=\tilde{M}(x, \hbar) \tilde{M}(x-\hbar, \hbar) \ldots \tilde{M}\left(x^{\prime}+\hbar, \hbar\right) M\left(x^{\prime}, \hbar\right),
$$

in the limit $\hbar \rightarrow 0$. Note that the matrices $\tilde{M}$ depend on the slowness parameter of the adiabatic change, $\hbar$, as well as the variable $x$. 
It is assumed that the matrices $\tilde{M}(x, \hbar)$ can be diagonalised

$$
\tilde{M}(x, \hbar)=\tilde{X}^{-1}(x, \hbar) \tilde{D}(x, \hbar) \tilde{X}(x, \hbar),
$$

where $\tilde{D}(x, h)$ is diagonal, and that all the eigenvalues are distinct and lie on the unit circle.

Now (3.1) can be written

$$
\tilde{G}\left(x, x^{\prime} ; \hbar\right)=\tilde{X}^{-1}(x, \hbar) \tilde{g}\left(x, x^{\prime} ; \hbar\right) \tilde{X}\left(x^{\prime}, \hbar\right)
$$

where

$$
\begin{aligned}
\tilde{\mathbf{g}}\left(x, x^{\prime} ; \hbar\right)=\tilde{D}(x, \hbar)[\tilde{1}+\hbar \tilde{V}(x-\hbar, \hbar)] \tilde{D}(x-\hbar, \hbar) \\
\\
\times[\tilde{\mathbf{1}}+\hbar \tilde{V}(x-2 \hbar, \hbar)] \ldots\left[\tilde{1}+\hbar \tilde{V}\left(x^{\prime}, \hbar\right)\right] \tilde{D}\left(x^{\prime}, \hbar\right),
\end{aligned}
$$

and

$$
\tilde{1}+\hbar \tilde{V}(x, \hbar)=\tilde{X}(x+\hbar, \hbar) \tilde{X}^{-1}(x, \hbar) .
$$

Throughout the calculation presented here, it will be sufficient to use the approximation

$$
\begin{aligned}
\tilde{V}(x) & =[\mathrm{d} \tilde{X}(x, \hbar) / \mathrm{d} x] \tilde{X}^{-1}(x, \hbar)+\mathrm{O}(\hbar) \\
& =[\mathrm{d} \tilde{X}(x, 0) / \mathrm{d} x] \tilde{X}^{-1}(x, 0)+\mathrm{O}(\hbar) .
\end{aligned}
$$

(The second equation of (3.6) shows that, when calculating $\tilde{V}$, the dependence of $\tilde{X}$ on $\hbar$ can be neglected and will not be shown in subsequent equations.) Now, using the notation

$$
\tilde{g}_{0}\left(x, x^{\prime} ; \hbar\right)=\tilde{D}(x, \hbar) \tilde{D}(x-\hbar, \hbar) \ldots \tilde{D}\left(x^{\prime}+\hbar, \hbar\right) \tilde{D}\left(x^{\prime}, \hbar\right),
$$

and ordering the expansion of equation (3.4) in powers of $\hbar$

$$
\tilde{g}\left(x, x^{\prime} ; \hbar\right)=\tilde{g}_{0}\left(x, x^{\prime} ; \hbar\right)+\hbar \sum_{\substack{n=0 \\ x^{\prime \prime}=x^{\prime}+n \hbar}}^{\sim} \tilde{g}_{0}\left(x, x^{\prime \prime}+\hbar\right) \tilde{V}\left(x^{\prime \prime}\right) \tilde{g}_{0}\left(x^{\prime \prime}, x^{\prime} ; \hbar\right)+\mathrm{O}\left(\hbar^{2} V^{2}\right),
$$

leads to an exact but implicit equation for $\tilde{\boldsymbol{g}}$

$$
\tilde{g}\left(x, x^{\prime} ; \hbar\right)=\tilde{g}_{0}\left(x, x^{\prime} ; \hbar\right)+\hbar \sum_{\substack{n=0 \\ x^{\prime \prime}=x^{\prime}+n \hbar}}^{N} \tilde{g}_{0}\left(x, x^{\prime \prime}+\hbar, \hbar\right) \tilde{V}\left(x^{\prime \prime}\right) \tilde{g}\left(x, x^{\prime} ; \hbar\right) \text {. }
$$

An asymptotic solution of (3.9) will now be sought in the form

$$
\tilde{g}\left(x, x^{\prime} ; \hbar\right)=\tilde{f}\left(x, x^{\prime}\right) \tilde{g}_{0}\left(x, x^{\prime} ; \hbar\right),
$$

where $\tilde{f}\left(x, x^{\prime}\right)$ is diagonal. This trial solution is an adiabatic approximation; it expresses the expectation that when $\hbar$ is small, so that $\tilde{M}$ varies slowly, an eigenvector $u_{i}\left(x^{\prime}\right)$ of $M\left(x^{\prime}, \hbar\right)$ becomes, upon multiplying by $G\left(x, x^{\prime} ; \hbar\right)$, the corresponding eigenvector $u_{i}(x)$ of $M(x, \hbar)$. Before going any further, it is useful to define diagonal matrices $\tilde{S}$ and $\tilde{v}$ as follows

$$
\begin{aligned}
& \tilde{D}(x, \hbar)=\exp \left[\mathrm{i} \tilde{S}^{\prime}(x, \hbar)\right] \\
& \tilde{S}\left(x, x^{\prime} ; \hbar\right)=\int_{x^{\prime}}^{x} \mathrm{~d} x^{\prime \prime} \tilde{S}^{\prime}\left(x^{\prime \prime}, \hbar\right), \\
& \tilde{v}_{i j}(x)=\left\{\begin{array}{cc}
\tilde{V}_{i j}(x) & i=j \\
0 & i \neq j .
\end{array}\right.
\end{aligned}
$$


Now, substituting (3.10) into (3.9), and making use of the definitions (3.11), (3.12)

$$
\begin{aligned}
g_{i j}\left(x, x^{\prime} ; \hbar\right)- & g_{0 i j}\left(x, x^{\prime} ; \hbar\right) \\
= & \hbar \sum_{x^{\prime \prime}} g_{0 i j}\left(x, x^{\prime \prime}+\hbar ; \hbar\right) V_{i j}\left(x^{\prime \prime}\right) g_{0 i j}\left(x^{\prime \prime}, x^{\prime} ; \hbar\right) f_{j}\left(x^{\prime \prime}, x^{\prime}\right) \\
= & \hbar \sum_{x^{\prime \prime}} \exp \left(\mathrm{i} \sum_{u=x^{\prime \prime}+n \hbar}^{x} S_{i}^{\prime}(u, \hbar)\right) V_{i j}\left(x^{\prime \prime}\right) f_{j}\left(x^{\prime \prime}, x^{\prime}\right) \exp \left(\mathrm{i} \sum_{u=x^{\prime}+n \hbar}^{x^{\prime \prime}} S_{j}^{\prime}(u, \hbar)\right) \\
= & \hbar \exp \left[\frac{1}{2} \mathrm{i}\left(S_{i}^{\prime}(x)+S_{j}^{\prime}\left(x^{\prime}\right)\right)\right] \sum_{x^{\prime \prime}} \exp \left[(\mathrm{i} / \hbar) S_{i}\left(x, x^{\prime \prime}+\hbar / 2 ; \hbar\right)\right] V_{i j}\left(x^{\prime \prime}\right) f_{j}\left(x^{\prime \prime}, x^{\prime}\right) \\
& \times \exp \left[(\mathrm{i} / \hbar) S_{j}\left(x^{\prime \prime}+\hbar / 2, x^{\prime} ; \hbar\right)\right]+\mathrm{O}(\hbar) \\
= & \exp \left[\frac{1}{2} \mathrm{i}\left(S_{i}^{\prime}(x)+S_{j}^{\prime}\left(x^{\prime}\right)\right)\right] \times \int_{x^{\prime}}^{x} \mathrm{~d} x^{\prime \prime} V_{i j}\left(x^{\prime \prime}\right) f_{j}\left(x^{\prime \prime}, x^{\prime}\right) \\
& \times \exp \left[(\mathrm{i} / \hbar)\left(S_{i}\left(x, x^{\prime \prime} ; \hbar\right)+S_{j}\left(x^{\prime \prime}, x^{\prime} ; \hbar\right)\right)\right]+\mathrm{O}(\hbar) .
\end{aligned}
$$

For terms with $i \neq j$, the integrand in (3.13) contains a rapidly oscillating term and gives a contribution of $\mathrm{O}(\hbar)$, whereas for $i=j$ it gives a finite contribution. Therefore

$$
\tilde{g}\left(x, x^{\prime} ; \hbar\right)=\tilde{g}_{0}\left(x, x^{\prime} ; \hbar\right)\left[\tilde{1}+\int_{x^{\prime}}^{x} \mathrm{~d} x^{\prime \prime} \tilde{f}\left(x^{\prime \prime}, x^{\prime}\right) \tilde{v}\left(x^{\prime \prime}\right)\right]+\mathrm{O}(\hbar),
$$

since only the diagonal elements of (3.13) remain. This justifies the use of the adiabatic approximation (3.10). From (3.10) and (3.14), $\tilde{f}$ satisfies

$$
\tilde{f}\left(x, x^{\prime}\right)=\tilde{1}+\int_{x^{\prime}}^{x} \mathrm{~d} x^{\prime \prime} \tilde{f}\left(x^{\prime \prime}, x^{\prime}\right) \tilde{v}\left(x^{\prime \prime}\right),
$$

which gives

$$
\tilde{f}\left(x, x^{\prime}\right)=\exp \left(\int_{x^{\prime}}^{x} \mathrm{~d} x^{\prime \prime} \tilde{v}\left(x^{\prime \prime}\right)\right)
$$

where both $\tilde{f}$ and $\tilde{v}$ are diagonal. Therefore the central result of this section, the formula for $\tilde{G}\left(x, x^{\prime} ; \hbar\right)$, is found to be

$$
\begin{aligned}
& \tilde{G}\left(x, x^{\prime} ; \hbar\right)=\tilde{X}^{-1}(x) \exp \left(\int_{x^{\prime}}^{x} \mathrm{~d} x^{\prime \prime} \tilde{v}\left(x^{\prime \prime}\right)\right) \tilde{g}_{0}\left(x, x^{\prime} ; \hbar\right) \tilde{X}\left(x^{\prime}\right)+\mathrm{O}(\hbar), \\
& \tilde{\mathbf{g}}_{0}\left(x, x^{\prime} ; \hbar\right)=\exp \left[\frac{1}{2} \mathrm{i}\left(\tilde{S}^{\prime}(x)+\tilde{S}^{\prime}\left(x^{\prime}\right)\right)\right] \exp \left[(\mathrm{i} / \hbar) S\left(x, x^{\prime} ; \hbar\right)\right]+\mathrm{O}(\hbar) .
\end{aligned}
$$

In this result the dependences of some quantities on $\hbar$ have not been shown, since they are not important at this order of accuracy.

The remainder of this section will discuss a slight simplification of (3.17) which is possible when the transfer matrices preserve some quantity $j$, which will be called the current. This is usually the case in one-dimensional quantum mechanical problems. For any two vectors $\phi, \psi$ the current $j$ is given by

$$
j_{\phi, \psi}=\phi^{* \top} \tilde{\boldsymbol{J}}
$$

(where $\tilde{J}$ is a constant matrix). If $j$ is preserved under the action of a transfer matrix $\tilde{M}$, then

$$
j_{M \phi, M \psi}=(\tilde{M} \phi)^{\top *} \tilde{J}(\tilde{M} \psi)=j_{\phi, \psi}
$$


so that $M$ satisfies

$$
\tilde{M}^{\mathrm{T} * \tilde{J} \tilde{M}}=\tilde{J}
$$

The transfer matrices introduced in $\$ 2$ will be shown later to have this property. It can be shown that properties of $\dot{M}$ that are preserved on multiplying matrices together are indeed preserved by the formula (3.17) for the product $\tilde{G}$; i.e. if it is real, unimodular, or satisfies the current conservation (3.20) then the approximate formula (3.17) for the product also has these properties.

It will be useful to get an expression for $\tilde{f}\left(x, x^{\prime}\right)$ in terms of the eigenvectors of $\tilde{M}(x, \hbar)$. Let $u_{i}(x)$ and $\boldsymbol{v}_{i}(x)$ be right and left eigenvectors of $\tilde{M}(x, \hbar)$ :

$$
\tilde{M} u_{i}=\lambda_{i} u_{i}, \quad v_{i} \tilde{M}=\lambda_{i} v_{i} \text {. }
$$

The $u_{i}$ are proportional to the columns of $\tilde{X}^{-1}$ and the $v_{i}$ to the rows of $\tilde{X}$, so that

$$
u_{i} \cdot v_{j}=N_{i} \delta_{i j}
$$

For matrices $\tilde{M}$ that satisfy (3.20), a useful relationship can be found connecting the left and right eigenvectors: from (3.20) it is easy to show that

$$
\left(\tilde{J}_{\boldsymbol{u}_{i}}\right)^{* \mathrm{~T}} \tilde{\boldsymbol{M}}=\left(\tilde{J} \boldsymbol{u}_{i}\right)^{* \mathrm{~T}} \lambda_{i}^{*-1}
$$

so that the eigenvalues and eigenvectors come in pairs, related by

$$
\lambda_{i^{\prime}}=\lambda_{i}^{*-1}, \quad \boldsymbol{v}_{i^{\prime}}=\left(\tilde{J}_{\boldsymbol{u}_{i}}\right)^{* \mathrm{~T}} \text {. }
$$

Since the eigenvalues $\lambda_{i}$ are all on the unit circle for the transfer matrices considered here,

$$
\boldsymbol{v}_{i}=\left(\tilde{J}_{\boldsymbol{u}_{i}}\right)^{* \mathrm{~T}}
$$

Now collecting equations $(3.16),(3.12),(3.6),(3.22),(3.25)$, a simple and useful formula can be given for $f_{i}\left(x, x^{\prime}\right)$, in terms of the eigenvector $\boldsymbol{u}_{i}(x)$

$$
\begin{aligned}
f_{i}\left(x, x^{\prime}\right)=\exp \left[\int_{x^{\prime}}^{x} \mathrm{~d} x^{\prime \prime}\left(d \tilde{X} /\left.\mathrm{d} x\right|_{x^{\prime \prime}} \tilde{X}^{-1}\left(x^{\prime \prime}\right)\right)_{i i}\right] \\
=\exp \left[-\int_{x^{\prime}}^{x} \mathrm{~d} x^{\prime \prime}\left(u^{* \mathrm{~T}} \tilde{J} \mathrm{~d} u / \mathrm{d} x\right) /\left.\left(u^{\mathrm{T} *} \tilde{J} u\right)\right|_{x^{\prime \prime}}\right] \\
=\exp \left[-\int_{x^{\prime}}^{x} \mathrm{~d} x^{\prime \prime} j_{u(\mathrm{~d} u / \mathrm{d} x)} /\left.j_{u u}\right|_{x^{\prime \prime}}\right]
\end{aligned}
$$

where $u=u_{i}\left(x^{\prime \prime}\right)$ is the $i$ th eigenvector of $M(x, \hbar)$.

\section{Solution of the wKB problem for Harper's equation}

This section uses the central results of $\S 3$, equations (3.17) and (3.26), to solve the problem of finding a satisfactory WKB theory for Harper's equation (2.1).

The WKB solution required is of the form of equation (1.1)

$$
\psi(x)=A(x) u(x) \exp \left(\frac{\mathrm{i}}{\hbar} \int^{x} p\left(x^{\prime}\right) \mathrm{d} x^{\prime}\right) .
$$

Locally, the solution can be described by a set of $q$ amplitudes, either amplitudes of 
a Bloch function $u_{n}$, or, equivalently, the Fourier components $a_{m}$ of the Bloch function, as in equation (2.5). Alternatively, in the Floquet picture, $\boldsymbol{\psi}$ and $u$ would be specified by just two amplitudes on a pair of adjacent lattice sites.

In the Floquet picture, by equation (3.17), if the wavefunction $\psi\left(x^{\prime}\right)$ is an eigenvector $u_{i}\left(x^{\prime}\right)$ at position $x^{\prime}$, then at $x$ it is given by

$$
\begin{aligned}
\psi(x) & =g_{i}\left(x, x^{\prime} ; \hbar\right) u_{i}(x) \\
& =f_{i}\left(x, x^{\prime}\right) g_{0 i}\left(x, x^{\prime} ; \hbar\right) u_{i}(x) .
\end{aligned}
$$

The $g_{0 i}\left(x, x^{\prime} ; \hbar\right)$ term in $(4.2)$, which can be written

$g_{0 i}\left(x, x^{\prime} ; \hbar\right)=\exp \left[\frac{1}{2} \mathrm{i}\left(S_{i}^{\prime}(x)+S_{i}^{\prime}\left(x^{\prime}\right)\right)\right] \exp \left((\mathrm{i} / \hbar) \int_{x}^{x^{\prime}} \mathrm{d} x^{\prime \prime} S_{i}^{\prime}\left(x^{\prime \prime}, \hbar\right)\right)$,

can be associated with the oscillatory term in (4.1), and the $f_{i}\left(x, x^{\prime}\right)$ term with the amplitude $A(x)$.

The derivative $S^{\prime}(x, h)$ of the 'action' $S\left(x, x^{\prime}, \hbar\right)$ therefore plays the role of the momentum $p$ of the phase space. The energy $E$ is given as a function of $x(=\delta)$ and $S^{\prime}(=k q)$ by $(2.6)$ and (2.7) in the Bloch picture, or alternatively by (2.14) in the Floquet picture. The first order term in $\hbar$ in the relations between $E, x$ and $S^{\prime}$ must be retained since $\hbar$ appears in the denominator of the argument of the exponential in (4.3).

It can be seen that the transfer matrix (2.10) satisfies the current conservation property (3.20), with

$$
\tilde{J}=\left(\begin{array}{cc}
0 & i \\
-i & 0
\end{array}\right)
$$

The current $j_{\phi . \psi}$ can equivalently be calculated for the corresponding $q$ dimensional vectors $|\phi\rangle,|\psi\rangle$

$$
j_{\phi, \psi}=\langle\phi|\hat{J}| \psi\rangle
$$

where in the direct representation (by means of the Bloch function, $u_{n}$ ) the matrix elements of $\tilde{J}$ are given by

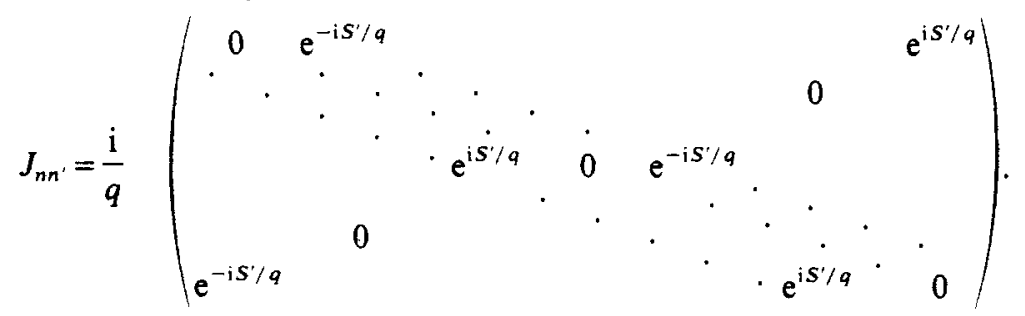

It is also useful to note that the eigenvalues and eigenvectors of the transfer matrices $\tilde{M}(x, E, h)$ come in complex conjugate pairs, corresponding to points related by $\pm S^{\prime}$ in the $x-S^{\prime}$ plane. The eigenvalues and eigenvectors are therefore real when $S^{\prime}=0$ :

$$
\boldsymbol{u}\left(x, S^{\prime}\right)=\boldsymbol{u}^{*}\left(x,-S^{\prime}\right) \text {. }
$$

If the eigenvectors $u_{i}$ are given as functions of $x$, then $A(x)$ for the solution (4.2) is given by (cf (3.26))

$$
A(x)=f_{i}\left(x, x^{\prime}\right)=\exp \left(-\int_{x^{\prime}}^{x} \mathrm{~d} x^{\prime \prime} j_{u(\mathrm{~d} u / \mathrm{d} x)} / j_{u u}\right)
$$


and is in general complex. Alternatively $A(x)$ could be chosen to be real; this condition then defines a connection rule for the phases of the eigenvector $\boldsymbol{u}(\boldsymbol{x})$. If the $\boldsymbol{u}(\boldsymbol{x})$ are given with some arbitrary phase, then the correct phase is established by means of a transformation

$$
u(x) \rightarrow u^{\prime}(x)=\exp (\mathrm{i} \phi(x)) u(x),
$$

so that the current matrix element $j_{u(d u / d x)}$ is transformed according to the equation

$$
j_{u(\mathrm{~d} u / \mathrm{d} x)} \rightarrow j_{u^{\prime}\left(\mathrm{d} u^{\prime} / \mathrm{d} x\right)}=j_{u(\mathrm{~d} u / \mathrm{d} x)}+\mathrm{i}(\mathrm{d} \phi / \mathrm{d} x) j_{u u} .
$$

Then by a suitable choice of $\phi(x), j_{u(d u / d x)}$ can be made to satisfy

$$
\operatorname{Im}\left(j_{u^{\prime}\left(\mathrm{d} u^{\prime} / \mathrm{d} x\right)}\right)=0,
$$

so that by (4.8), $A(x)$ is now real.

\section{The Bohr-Sommerfeld quantisation rule}

This section derives the Bohr-Sommerfeld quantisation rule (1.3), which is the condition for single-valuedness of the WKB solution under continuation around a closed phase trajectory in the $x, S^{\prime}$ plane.

In this section it will be useful to consider the eigenvector $u^{\prime}\left(x, S^{\prime}\right)$ to be a given single-valued function defined on the phase plane. If $\boldsymbol{A}(\boldsymbol{x})$ is to be a real function, the eigenvector $\boldsymbol{u}^{\prime}$ must be multiplied by a phase factor so that the modified eigenvector $u\left(x, S^{\prime}\right)$ satisfies the connection formula (4.11), i.e.

$$
\operatorname{Im}\left[\left(j_{u(\lambda u / \partial x)}\right) \Delta x+\left(j_{u\left(\lambda u / \lambda S^{\prime}\right)}\right) \Delta S^{\prime}\right]=\operatorname{Im}\left(j_{u \nabla u}\right) \cdot \Delta X=0,
$$

for transport of $u$ by a vector $\Delta X=\left(\Delta x, \Delta S^{\prime}\right)$ in the phase plane. This connection (5.1) is non-holonomic, and on transporting $\boldsymbol{u}$ around a closed circuit $C$ in phase space, it is multiplied by a phase factor $\mathrm{e}^{\mathrm{i} \gamma_{c}}$, given by

$$
\begin{aligned}
\gamma_{c} & =\operatorname{Im} \oint_{c}\left(j_{\mathcal{u}^{\prime} \nabla u^{\prime}} / j_{u^{\prime} u^{\prime}}\right) \cdot \mathrm{d} \boldsymbol{X} \\
& =\operatorname{Im} \oint_{c} j_{u^{\prime}\left(\mathrm{d} u^{\prime} / \mathrm{d} x\right)} / j_{u^{\prime} u^{\prime}} \mathrm{d} x .
\end{aligned}
$$

On transporting $\boldsymbol{u}$ around a phase trajectory of energy $E$, there is thus a phase change $\mathrm{e}^{\mathrm{i} \gamma(E)}$, where

$$
\begin{aligned}
\gamma(E) & =\operatorname{Im} \oint_{\varepsilon=E}\left(j_{u^{\prime} \nabla u^{\prime}} / j_{u^{\prime} u^{\prime}}\right) \cdot \mathrm{d} \boldsymbol{X} \\
& =\operatorname{Im} \oint_{\varepsilon=E} j_{u^{\prime}\left(\mathrm{d} u^{\prime} / \mathrm{d} x\right) / j_{u^{\prime} u^{\prime}} \mathrm{d} x .}
\end{aligned}
$$

This phase change makes a contribution to the Bohr-Sommerfeld quantisation formula.

In order to describe correctly the continuation of the solution around the phase trajectory of energy $E$, it is necessary to consider carefully what happens at the classical turning points, where $S^{\prime}=0 \bmod 2 \pi$. On the lines $S^{\prime}=0$, the current $j_{u u}$ is zero, since by $(4.7), u(x, 0)$ is real. In the neighbourhood of the line $S^{\prime}=0, j_{u u}\left(x, S^{\prime}\right)$ takes the form

$$
j_{u \text { u }}=a(x) S^{\prime}+\mathrm{O}\left(S^{\prime 2}\right) \text {, }
$$


for some function $a(x)$. Now consider the form of $j_{u \nabla u}$ near the line $S^{\prime}=0$. Consider the result

$$
j_{u+\Delta u, u+\Delta u}=j_{u u}+2 \operatorname{Re} j_{u, \Delta u}+\mathrm{O}\left(\Delta u^{2}\right)
$$

(which uses the fact that the current operator is Hermitian), and take $\boldsymbol{u}=\boldsymbol{u}(x, 0)$ and $\boldsymbol{u}+\Delta \boldsymbol{u}=\boldsymbol{u}\left(x+\Delta x, \Delta S^{\prime}\right)$. Then using (5.5) and ignoring $\mathrm{O}\left(\Delta u^{2}\right)$

$$
j_{u+\Delta u, u+\Delta u}=2 \operatorname{Re} j_{u \Delta u}=2 \operatorname{Re} j_{u \nabla u} \cdot \Delta X=a(x+\Delta x) \Delta S^{\prime}
$$

so that, near $S^{\prime}=0$

$$
\operatorname{Re}\left(j_{u \nabla u}\right)=\left(\mathrm{O}, \frac{1}{2} a(x)\right)+\mathrm{O}(x)+\mathrm{O}\left(S^{\prime}\right) .
$$

Now, from (4.8), the amplitude $A(x)$ (constrained to be real) is given by

$$
A(x)=\exp \left(-\left.\operatorname{Re} \int^{x}\left[j_{u(\mathrm{~d} u / \mathrm{d} x)} / j_{u u}\right]\right|_{x^{\prime}} \mathrm{d} x^{\prime}\right) .
$$

Near the line $S^{\prime}=0$, therefore,

$$
\begin{aligned}
& A(x) \simeq \exp \left[\int^{x}-\frac{1}{2} a\left(x^{\prime}\right) /\left(a\left(x^{\prime}\right) S^{\prime}\left(x^{\prime}\right)\right) \cdot\left(\mathrm{d} S^{\prime} / \mathrm{d} x^{\prime}\right) \mathrm{d} x^{\prime}\right] \\
& A(x) \simeq \text { constant }\left[S^{\prime}(x)\right]^{-1 / 2} .
\end{aligned}
$$

Thus $A(x)$ diverges at a classical turning point, $x_{0}$, where $S^{\prime}=0$. Near this point the form of the phase trajectory is given by

$$
S^{2}=\operatorname{constant}\left(x-x_{0}\right)
$$

so that as $x_{0}$ is approached from within the classically allowed region, $A(x)$ diverges as

$$
A(x) \sim \text { constant }\left(x-x_{0}\right)^{-1 / 4} .
$$

(Of course there is not a real divergence of the exact solution, only in the WKB approximation; the assumption used in $\S 3$ that the eigenvalues of the transfer matrix are distinct breaks down when $S^{\prime}=0$.) The divergence of $A(x)$ given by (5.11) is of exactly the same type as is encountered in ordinary шкв problems at first-order turning points, and any of the usual arguments (e.g. continuation in the complex plane, see Landau and Lifshitz (1958)) show that an extra phase change of $\pi / 2$ must be included for each of the two classical turning points of the phase trajectory.

The final contribution to the phase change of $\psi(x)$ is from the phase integral term: this is

$$
\frac{1}{\hbar} \oint_{\varepsilon\left(x, S^{\prime}\right)=E} S^{\prime}(x, \hbar) \mathrm{d} x .
$$

As noted earlier, the correction to $\varepsilon\left(x, s^{\prime}\right)$ of first order in $\hbar$ must be retained when evaluating (5.12), since $\hbar$ appears in the denominator. Collecting together all these contributions to the phase gives the Bohr-Sommerfeld quantisation rule for the system.

$$
2 \pi n=\frac{1}{\hbar} \oint_{\varepsilon\left(x, S^{\prime}\right)=E} S^{\prime}(x, \hbar) \mathrm{d} x+\pi+\gamma(E) .
$$

Finally there are two important points which must be mentioned. Firstly, because $j_{u u}$ is zero on the line $S^{\prime}=0$, the integrand in the formula for $\gamma(E)$ diverges at the 
classical turning point as $\left(x-x_{0}\right)^{-1 / 2}$. The integral $\gamma(E)$ remains finite, but does not tend to zero as $E$ approaches a maximum or minimum of $\varepsilon\left(x, S^{\prime}\right)$, and the phase trajectory shrinks to a point. Instead, $\gamma(E)$ tends to a finite limit $\gamma_{0}$ at the top or bottom of a band. This limiting value of $\gamma(E)$ at the band edges is calculated in the appendix.

Secondly, there are some special cases which should be mentioned. When the rational number $p / q$ to which $\beta$ approximates is zero, then both the phase $\gamma(E)$ and the $\hbar$ dependent corrections to $\varepsilon\left(x, S^{\prime}\right)$ vanish, and the Bohr-Sommerfeld quantisation condition takes the usual form. This case is discussed in detail in Wilkinson (1984). There are also some simplifications which occur when $p / q=\frac{1}{2}$, and it is only for $p / q$ with denominators greater than two that all the effects described in this paper are seen.

\section{Summary and comparison with numerical results}

In this section some comparisons will be made of eigenvalues calculated using the Bohr-Sommerfeld quantisation rule with those calculated exactly. Firstly, however, the important formulae are collected together and summarised.

The parameter $\beta$ in equation (2.1) is written

$$
\beta=\beta_{0}+\Delta \beta=p / q+\hbar / 2 \pi q
$$

The energy $E$ is considered to be a function $\varepsilon\left(x, S^{\prime}\right)$ of the phase plane coordinates $x$ and $S^{\prime}$. The relationship between $E, x$ and $S^{\prime}$ is, in the Bloch picture, given by the eigenvalue equation

$$
\hat{H}\left(x, S^{\prime}\right)|u\rangle=E|u\rangle,
$$

and the matrix elements of $\hat{H}$ are given by (2.7), with $\delta \rightarrow x$ and $k \rightarrow S^{\prime} / q$ :

$$
\begin{aligned}
& H_{n n^{\prime}}\left(x, S^{\prime}\right)
\end{aligned}
$$

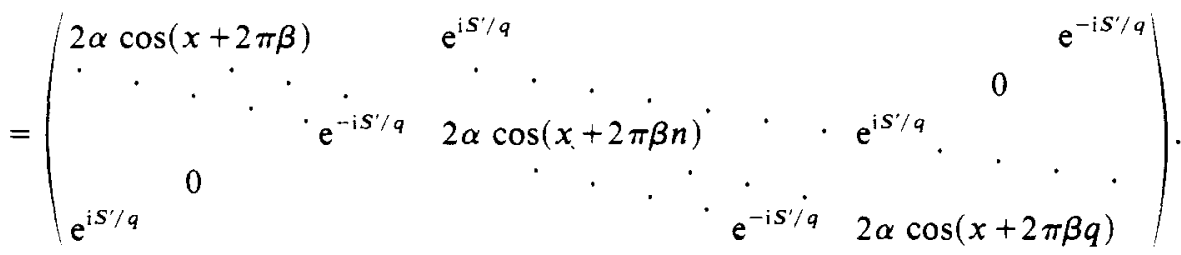

Equivalently, in the Floquet picture, this relationship is given by

$$
2 \cos S^{\prime}=\operatorname{Tr} \tilde{M}_{E}(x, \hbar)
$$

where

$$
\begin{gathered}
\tilde{M}_{E}(x, \hbar)=\tilde{T}(x+2 \pi \beta(q-1), E) \ldots \tilde{T}(x+2 \pi \beta, E) \tilde{T}(x, E), \\
\tilde{T}(x, E)=\left(\begin{array}{cc}
E-2 \alpha \cos x & -1 \\
1 & 0
\end{array}\right) .
\end{gathered}
$$

The phase change $\gamma(E)$ is given by a line integral in phase-space around a phase trajectory

$$
\gamma(E)=\operatorname{Im} \oint_{\varepsilon\left(x, S^{\prime}\right)=E}\left(j_{u \nabla u} / j_{u u}\right) \cdot \mathrm{d} \boldsymbol{X} .
$$


In the Bloch picture, the vector $u\left(x, S^{\prime}\right)$ is an eigenvector of $\hat{H}\left(x, S^{\prime}\right)$, given by (6.3), and in the Floquet picture $u\left(x, S^{\prime}\right)$ is an eigenvector of the transfer matrix $\tilde{M}\left(x, \varepsilon\left(x, S^{\prime}\right), \hbar\right)$. The matrix elements of the current operator are, in the Bloch picture

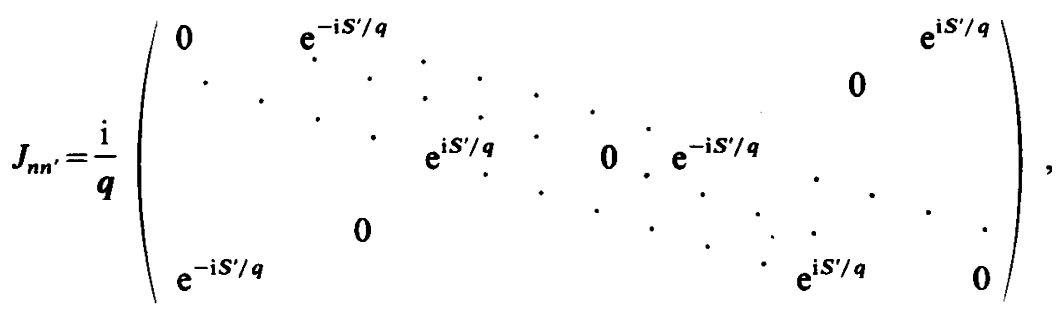

and in the Floquet picture

$$
\tilde{J}=\left(\begin{array}{cc}
0 & \mathrm{i} \\
-\mathrm{i} & 0
\end{array}\right)
$$

The final result, the Bohr-Sommerfeld quantisation condition, is (cf 5.13)

$$
\oint_{\varepsilon=E} S^{\prime}(x, E, \hbar) \mathrm{d} x=2 \pi\left[n+\frac{1}{2}-(1 / 2 \pi) \operatorname{sign}(\hbar) \gamma(E)\right] \cdot|\hbar|
$$

(remember that $\hbar$ can be negative for this system).

Now the theoretical predictions of this paper will be compared with some numerical results.

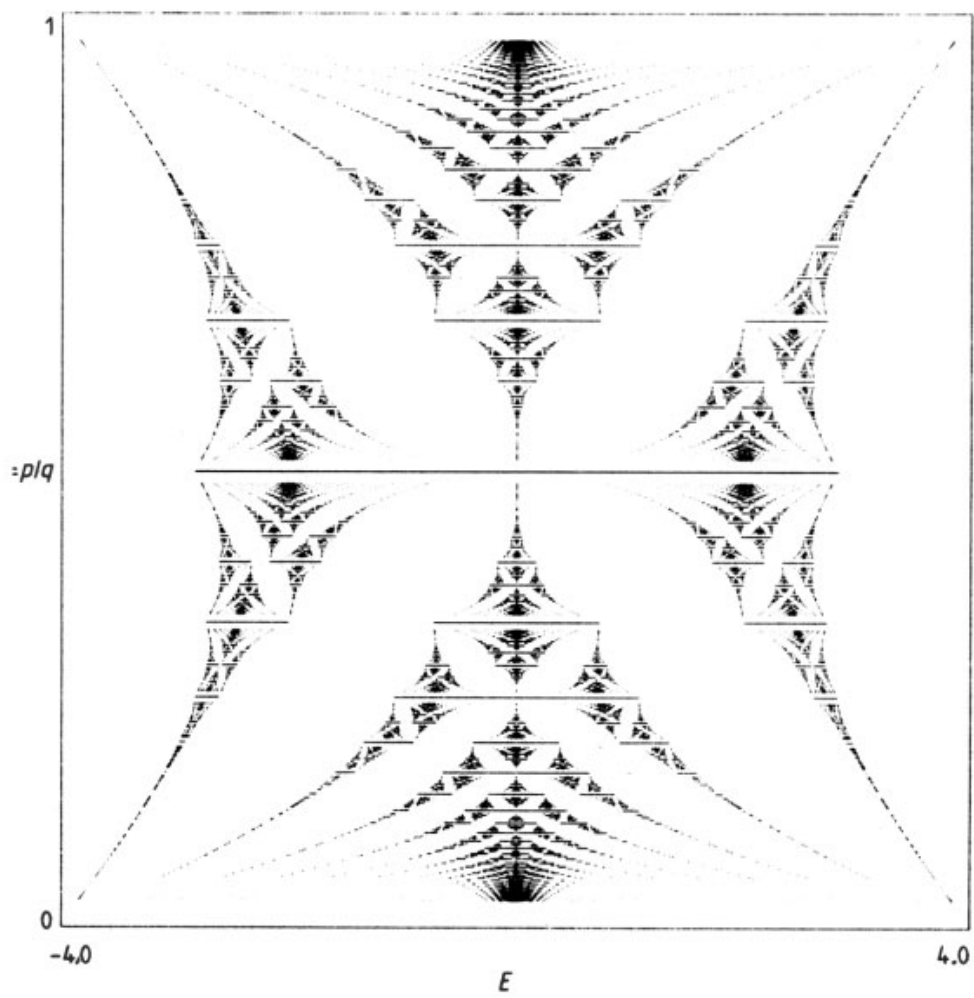

Figure 3. A plot of the spectrum of Harper's equation, plotted for every rational $\beta=p / q$ with $q \leqslant 40$. This picture illustrates the situation shown schematically in figure 2 . 
First, figure 3 gives a general illustration of the ideas discussed in this paper. This picture, originally published by Hofstadter (1976), is a plot of the spectrum (i.e. the support of $\varepsilon(\delta, k)$ ) of Harper's equation for every rational value of $\beta$ with denominator $q$ less than 40 . There are $q$ energy bands (the central two touching when $q$ is even). When $\beta$ is close to a low-denominator rational, $p_{0} / q_{0}$, then the $q$ bands are very narrow and cluster into $q_{0}$ groups, which correspond to the $q_{0}$ bands when $\beta=\beta_{0}=p_{0} / q_{0}$. These $q$ narrow bands are the Bohr-Sommerfeld levels discussed in this paper, slightly broadened by tunnelling effects.

It is also easy to see by inspection of figure 3 that, except for $\beta_{0}=0,1$ and $\beta_{0}=\frac{1}{2}$, the Bohr-Sommerfeld quantisation is not of the usual form, i.e. action $=2 \pi(n+1 / 2) \hbar$, since the pattern is not symmetrical about and below the lines $\beta=\beta_{0}$ (cf equation (6.9), which is not symmetric under $\hbar \rightarrow-\hbar$ ).

Next, table 1 gives some comparisons of energy levels predicted using formulae (6.1)-(6.9), $E_{\mathrm{pr}}$, with exact levels, $E_{\text {ex }}$ obtained by the same method as Hofstadter, using high precision arithmetic. In all cases except one the broadening of these levels by tunnelling is smaller than the six-digit precision of the eigenvalues $E_{\mathrm{ex}}$. The meaning of the columns $p_{0}, q_{0}$, is given by equation (6.1), $n$ is the quantum number of the Bohr quantised level (as in equation (6.9)) and $n_{b}$ labels the energy band within which this level lies. The subscripts $t$ and $b$ of $n_{b}$ mean that $n$ is counted from the top and bottom of the energy band respectively. Finally, $\Delta E$ is the separation of the closest neighbouring energy level, and gives a scale against which the error $\left|E_{\mathrm{ex}}-E_{\mathrm{pr}}\right|$ of the predictions should be compared.

Finally, table 2 shows how the error in the predicted levels decreases very rapidly as $\hbar$ decreases. These results suggest that the error of the prediction is $O\left(\hbar^{3}\right)$ compared to the separation of levels which decreases as $\mathrm{O}(\hbar)$.

Table 1. Comparison of predicted energy levels $E_{\mathrm{pr}}$ with exact levels $E_{\mathrm{ex}}$. For full description see $\$ 6$ of text. For all values in this table, $\alpha=1$.

\begin{tabular}{llllllllll}
\hline$p_{0}$ & $q_{0}$ & $\Delta \beta$ & $n_{\mathrm{b}}$ & $n$ & \multicolumn{1}{c}{$E_{\mathrm{pr}}$} & $E_{\mathrm{ex}}$ & $\left|E_{\mathrm{pr}}-E_{\mathrm{ex}}\right|$ & $\Delta E$ & $\gamma(E)$ \\
\hline 1 & 3 & $1 / 200$ & $1 \mathrm{t}$ & 1 & -1.99435 & -1.99277 & 0.00158 & 0.08395 & 1.6791 \\
1 & 3 & $1 / 200$ & $1 \mathrm{t}$ & 6 & -2.29301 & -2.29209 & 0.00092 & 0.04154 & 0.6868 \\
1 & 3 & $1 / 200$ & $1 \mathrm{t}$ & 10 & -2.40938 & -2.40860 & 0.00078 & 0.02295 & -0.4313 \\
1 & 3 & $1 / 200$ & $2 \mathrm{~b}$ & 1 & -0.61889 & -0.62003 & 0.00114 & 0.10129 & -1.6406 \\
1 & 3 & $1 / 200$ & $3 \mathrm{t}$ & 1 & 2.70954 & 2.71090 & 0.00136 & 0.03379 & -2.2567 \\
1 & 3 & $1 / 200$ & $3 \mathrm{t}$ & 5 & 2.58201 & 2.58442 & 0.00241 & 0.02839 & -3.5757 \\
1 & 4 & $1 / 300$ & $2 \mathrm{t}$ & 1 & -0.28742 & -0.28171 & 0.00621 & 0.10782 & 1.4364 \\
1 & 4 & $1 / 300$ & $2 \mathrm{t}$ & 5 & -0.58727 & -0.58578 & 0.00148 & 0.04299 & 0.0375 \\
1 & 3 & $2 / 387$ & $1 \mathrm{t}$ & 1 & -1.99414 & -1.99252 & 0.00162 & 0.08631 & 1.6786 \\
1 & 3 & $2 / 387$ & $1 \mathrm{t}$ & 7 & -2.33540 & -2.33427 to & 0.001 & 0.035 & 0.4193 \\
& & & & & & -2.33432 & & & -0.5570 \\
3 & 7 & $1 / 1960$ & $3 \mathrm{~b}$ & 1 & -1.58070 & -1.58068 & 0.00002 & 0.00623 & -0.630 \\
3 & 7 & $1 / 1960$ & $3 \mathrm{~b}$ & 5 & -1.60464 & -1.60460 & 0.00004 & 0.00557 & -0.6380 \\
\hline
\end{tabular}

\section{Concluding remarks}

This paper has demonstrated a novel type of Bohr-Sommerfeld quantisation, involving a non-holonomic connection rule for transporting the eigenvectors $\boldsymbol{u}$ of the matrixvalued Hamiltonian function $\hat{H}(x, p)$ around a circuit in phase space. 
Table 2. Illustrating the rapid improvement of the predictions as $\hbar$ decreases. All the results in this table refer to the case $\alpha=1, p_{0}=1, q_{0}=3, n_{\mathrm{b}}=3 t$. Using equation (A9), the limiting value of $\gamma(E)$ at the edge of the band concerned is predicted to be $\gamma_{0}=-1.8200$. The values of $\gamma(E)$ for the $n=1$ states approach this limiting value.

\begin{tabular}{lllllll}
\hline$n$ & $\Delta \beta$ & $E_{\mathrm{pr}}$ & $E_{\mathrm{ex}}$ & $\left|E_{\mathrm{pr}}-E_{\mathrm{ex}}\right|$ & $\Delta E$ & $\gamma(E)$ \\
\hline 1 & $1 / 100$ & 2.68295 & 2.69043 & 0.00748 & 0.06637 & -2.9737 \\
& $1 / 200$ & 2.70954 & 2.71090 & 0.00136 & 0.03379 & -2.2567 \\
& $1 / 400$ & 2.72120 & 2.72140 & 0.00020 & 0.01710 & -1.9760 \\
& $1 / 800$ & 2.72671 & 2.72671 & 0.00000 & 0.00859 & -1.8554 \\
5 & $1 / 200$ & 2.58201 & 2.58442 & 0.00241 & 0.02839 & -3.5757 \\
& $1 / 400$ & 2.65491 & 2.65514 & 0.00023 & 0.01575 & -2.3862 \\
& $1 / 800$ & 2.69286 & 2.69288 & 0.00002 & 0.00826 & -2.0428 \\
\hline
\end{tabular}

The phase change $\gamma$ of the eigenvector has been determined in terms of the line integral of the connection (5.2). In principle, $\gamma$ could also be expressed as the integral of the curvature of the connection over the area enclosed by $C$. In practice, however, this is not useful, since this curvature is singular on the line $S^{\prime}=0$, and in any case for a computer calculation of $\gamma(E)$ the line integral is much easier to evaluate.

The method given in this paper is easily adapted to the problem of determining the Bohr-Sommerfeld quantisation condition for Bloch electrons in a weak magnetic field. It is well known that this condition takes the form

$$
\mathscr{A}_{k}=2 \pi(e B / \hbar)(n+\Gamma),
$$

where $\mathscr{A}_{k}$ is the area enclosed by a section through the Fermi surface perpendicular to the magnetic field (Onsager 1952). The constant $\Gamma$ is not determined by Onsager's argument, and has previously only been determined exactly by very elaborate methods based on the effective Hamiltonian approach (see e.g. Roth 1966). The main result is that, for crystals with centres of inversion, $\Gamma$ is always equal to $\frac{1}{2}$ (plus terms of higher order in the magnetic field), but when there is not a centre of inversion there is an additional component of $\Gamma$ given by an integral analogous to (5.3).

It is worthwhile to note that two other results have appeared recently which involve a non-holonomic connection rule for the phase of the eigenvector of a matrix.

Firstly Mead and Truhlar (1979) and Berry (1984) calculate the phase change of the wavefunction of a system after being varied slowly around a cyclic path in the space of some parameters of the system. In terms of the matrix product calculation of $\S 3$ of this paper, this corresponds to considering a string of slowly varying unitary evolution operators, i.e. to the case $\tilde{J}=\tilde{1}$ in equation (3.20).

Secondly Thouless et al (1982) have considered the quantised Hall effect in samples with a weak periodic potential with a rational number of flux quanta per unit cell. They show that the Hall conductance of a full sub-band is $e^{2} / 2 \pi h$ times the phase change when the wavefunction is transported around the edge of the magnetic Brillouin zone using the adiabatic connection rule of Mead and Berry. Because the magnetic Brillouin zone is topologically a torus, they are able to show that this phase change is $2 \pi$ times an integer. Simon (1983) has exhibited a connection between the work of Berry and Mead, and that of Thouless $e t$ al, and has emphasised the importance of the idea of a non-holonomic connection.

The formula given in $\S 3$ for the product of a string of slowly varying matrices is a very general result, and may have many uses other than those considered here. 


\section{Acknowledgment}

I wish to thank the UK Science and Engineering Research Council for a postgraduate studentship.

\section{Appendix}

This appendix demonstrates the result that $\gamma(E)$ approaches a finite limit $\gamma_{0}$ at the top or bottom of an energy band, by calculating this limit in terms of the transfer matrices.

The transfer matrix is assumed to be known in the form of a series expansion:

$$
\begin{aligned}
\tilde{M}(x, E, \hbar)= & \left(\begin{array}{ll}
A & B \\
C & D
\end{array}\right)=\left(\begin{array}{ll}
A_{0} & B_{0} \\
C_{0} & D_{0}
\end{array}\right) \\
& +\left(x-x_{0}\right)\left(\begin{array}{ll}
a_{x} & b_{x} \\
c_{x} & d_{x}
\end{array}\right)+\left(E-E_{0}\right)\left(\begin{array}{ll}
a_{E} & b_{E} \\
c_{E} & d_{E}
\end{array}\right)+\hbar\left(\begin{array}{ll}
a_{\hbar} & b_{\hbar} \\
c_{\hbar} & d_{\hbar}
\end{array}\right)+\ldots,
\end{aligned}
$$

where $x_{0}$ and $E_{0}$ are the values of $x$ and $E$ corresponding to the top or bottom of the energy band $E=\varepsilon\left(x, s^{\prime}\right)$ when $\hbar=0$. Also, it is assumed that the first few coefficients in the expansion of $\operatorname{Tr} \tilde{M}$ are known

$$
2 \cos S^{\prime}=\operatorname{Tr} \tilde{M}(x, E, \hbar)=2+r\left(x-x_{0}\right)^{2}-s\left(E-E_{0}\right)-t \hbar .
$$

From equation (2.8), it can be seen that $r=q^{2} \alpha^{q}$. From (A2), the momentum $S^{\prime}$ is given by

$$
S^{\prime 2}=s\left(E-E_{0}\right)-r\left(x-x_{0}\right)^{2}-t \hbar
$$

in the neighbourhood of the band edge. The action $S(E)$ for the phase trajectory of energy $E$ is given by

$$
S(E)=\oint_{\varepsilon=E} S^{\prime} \mathrm{d} x=\left(\pi / \alpha^{q} q\right)\left[s\left(E-E_{0}\right)-t \hbar\right]
$$

Now the phase change $\gamma(E)$ of the eigenvector $\boldsymbol{u}$ of $\tilde{M}$ will be found, in the neighbourhood of the energy $E_{0}$. The transfer matrix $\tilde{M}$ of (A1) can be diagonalised as follows

$$
\begin{array}{ll}
\tilde{M}(x, E, \hbar)=\tilde{X}^{-1} \tilde{D} \tilde{X}, & \tilde{D}=\left(\begin{array}{cc}
\mathrm{e}^{\mathrm{i} S^{\prime}} & 0 \\
0 & \mathrm{e}^{-\mathrm{i} S^{\prime}}
\end{array}\right) \\
\tilde{X}=\left(\begin{array}{cc}
C & D-\mathrm{e}^{-\mathrm{i} S^{\prime}} \\
-C & \mathrm{e}^{\mathrm{i} S^{\prime}}-D
\end{array}\right), & \tilde{X}^{-1}=\frac{1}{2 \mathrm{i} C \sin S^{\prime}}\left(\begin{array}{cc}
\mathrm{e}^{\mathrm{i} S^{\prime}}-D & \mathrm{e}^{-\mathrm{i} S^{\prime}}-D \\
C & C
\end{array}\right) .
\end{array}
$$

Now, for the eigenvector corresponding to the eigenvalue $\mathrm{e}^{\mathrm{i} S^{\prime}}$, the differential element of the phase change $\gamma$ is (retaining only lowest order terms)

$$
\mathrm{d} \gamma_{1}=\operatorname{Im} \mathrm{d} \tilde{V}_{11}=\operatorname{Im}\left(\mathrm{d} \tilde{X} \tilde{X}^{-1}\right)_{11}=\frac{D-1}{2 C S^{\prime}} \mathrm{d} C-\frac{\mathrm{d} D}{2 S^{\prime}} .
$$


Using the relationship (A3) between $x, S^{\prime}$ and $E$, for any quantity $A=A(x, E)$

$$
\left(\frac{\partial A}{\partial S^{\prime}}\right)_{x}=\frac{2 S^{\prime}}{s}\left(\frac{\partial A}{\partial E}\right)_{x}, \quad\left(\frac{\partial A}{\partial x}\right)_{S^{\prime}}=\left(\frac{\partial A}{\partial x}\right)_{E}+\frac{2 \alpha^{q} q^{2}\left(x-x_{0}\right)}{s}\left(\frac{\partial A}{\partial E}\right)_{x} .
$$

Using (A7) in (A6), to lowest order

$$
\begin{aligned}
& \mathrm{d} \gamma_{1}=\gamma_{x} \mathrm{~d} x+\gamma_{S^{\prime}} \mathrm{d} S^{\prime}, \\
& \gamma_{x}=\left\{\left[\left(D_{0}-1\right) / 2 C_{0}\right] c_{x}-\frac{1}{2} \mathrm{~d}_{x}\right\} / S^{\prime}+\mathrm{O}(1)=\kappa / S^{\prime}+\mathrm{O}(1), \\
& \gamma_{S^{\prime}}=\mathrm{O}(1) .
\end{aligned}
$$

Also, it is easy to show that $\mathrm{d} \gamma_{2}\left(x,-S^{\prime}\right)=\mathrm{d} \gamma_{1}\left(x, S^{\prime}\right)$. The phase change is then given by, in the limits $x, S^{\prime}, \hbar \rightarrow 0$ :

$$
\gamma(E) \simeq \gamma_{0}=\oint_{\varepsilon=E} \gamma_{x} \mathrm{~d} x+\gamma_{S^{\prime}} \mathrm{d} S^{\prime} \simeq \oint_{\kappa / S^{\prime} \mathrm{d} x}
$$

i.e.

$$
\gamma_{0}=2 \pi \kappa / \alpha^{q / 2} q, \quad \kappa=\left[\left(D_{0}-1\right) / 2 C_{0}\right] c_{x}-\frac{1}{2} \mathrm{~d}_{x} .
$$

The Bohr-Sommerfeld quantisation condition in the neighbourhood of the band edge then becomes (using (5.13), (A4), (A9))

$$
E=E_{0}+(1 / s) \cdot 2 \alpha^{q} q \hbar\left[n+\frac{1}{2}-\left(\kappa / \alpha^{q / 2} q\right) \operatorname{sign}(\hbar)+t / 2 \pi\right],
$$

where the level number satisfies the inequality

$$
n>\left(\kappa / \alpha^{q / 2} q\right) \cdot \operatorname{sign}(\hbar)-\frac{1}{2}
$$

\section{References}

Bellissard J and Simon B 1982 J. Funct. Anal. $48408-19$

Berry M V 1984 Proc. R. Soc. A392 45-57

Harper P G 1955 Proc. Phys. Soc. A68 874-8

Hofstadter D R 1976 Phys. Rev. B14 2239-49

Khinchin A Ya 1964 Continued Fractions (Chicago: University Press)

Landau L D and Lifshitz E M 1958 Quantum Mechanics ch 7 (Oxford: Pergamon)

Mead C A $1979 \mathrm{~J}$. Chem. Phys. 70 2276-83

Mead C A and Truhlar D G 1979 J. Chem. Phys. 70 2284-96

Onsager L 1952 Phil. Mag. 43 1006-8

Roth L M 1966 Phys. Rev. $145434-48$

Simon B 1982 Adv. Appl. Maths. 3 463-90

1983 Phys. Rev. Leti. 51 2167-70

Sokoloff J B 1981 Phys. Rev. B23 2039-41

Thouless D J, Kohmoto M, Nightingale M P and den Nijs M 1982 Phys. Rev. Lett. 49 405-9

Wilkinson M 1984 Proc. R. Soc. A391 305-50 